Supporting Information

\title{
Waved 2D Transition-Metal Disulfides for Nanodevices and Catalysis: A First-Principle Study
}

Youchao Kong ${ }^{\dagger}$, Haoqiang Ai*, Wei Wang ${ }^{\dagger}$, Xiuhua Xie ${ }^{\dagger}$ ๆ, Kin Ho Lo*, Shuangpeng Wang ${ }^{\dagger,}$ * $^{*}$, and Hui

$\operatorname{Pan}^{\dagger, \S *}$

${ }^{\dagger}$ MOE Joint Key Laboratory, Institute of Applied Physics and Materials Engineering, University of Macau, Macao SAR, 999078, P. R. China

tDepartment of Electromechanical Engineering, Faculty of Science and Technology, University of Macau, Macao SAR, 999078, P. R. China

\State Key Laboratory of Luminescence and Applications, Changchun Institute of Optics, Fine

Mechanics and Physics, Chinese Academy of Sciences, Changchun, 130033, P. R. China

$\S$ Department of Physics and Chemistry, Faculty of Science and Technology, University of Macau,

Macao SAR, 999078, P. R. China

\section{Corresponding Authors}

Hui Pan: huipan@um.edu.mo (email),+853 88224427 (tel.),+853 88222454 (fax).

Shuangpeng Wang: spwang@um.edu.mo (email). 


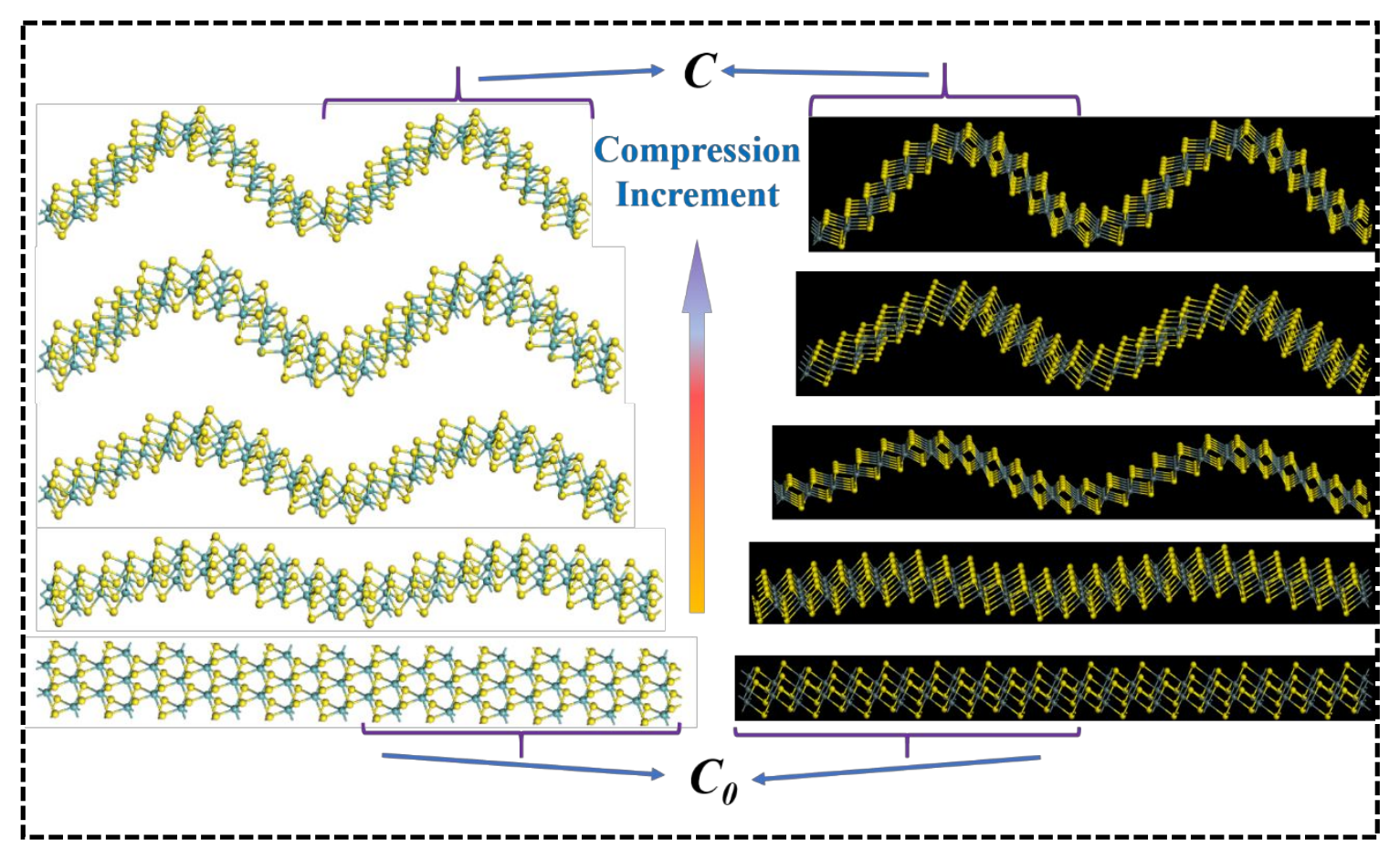

Figure S1. Representative structures of $2 \mathrm{H}$ (left column) and 1T (right column)

$\mathrm{p}-\mathrm{MS}_{2}$ and $\mathrm{w}-\mathrm{MS}_{2}$ with different compressions in the armchair direction.

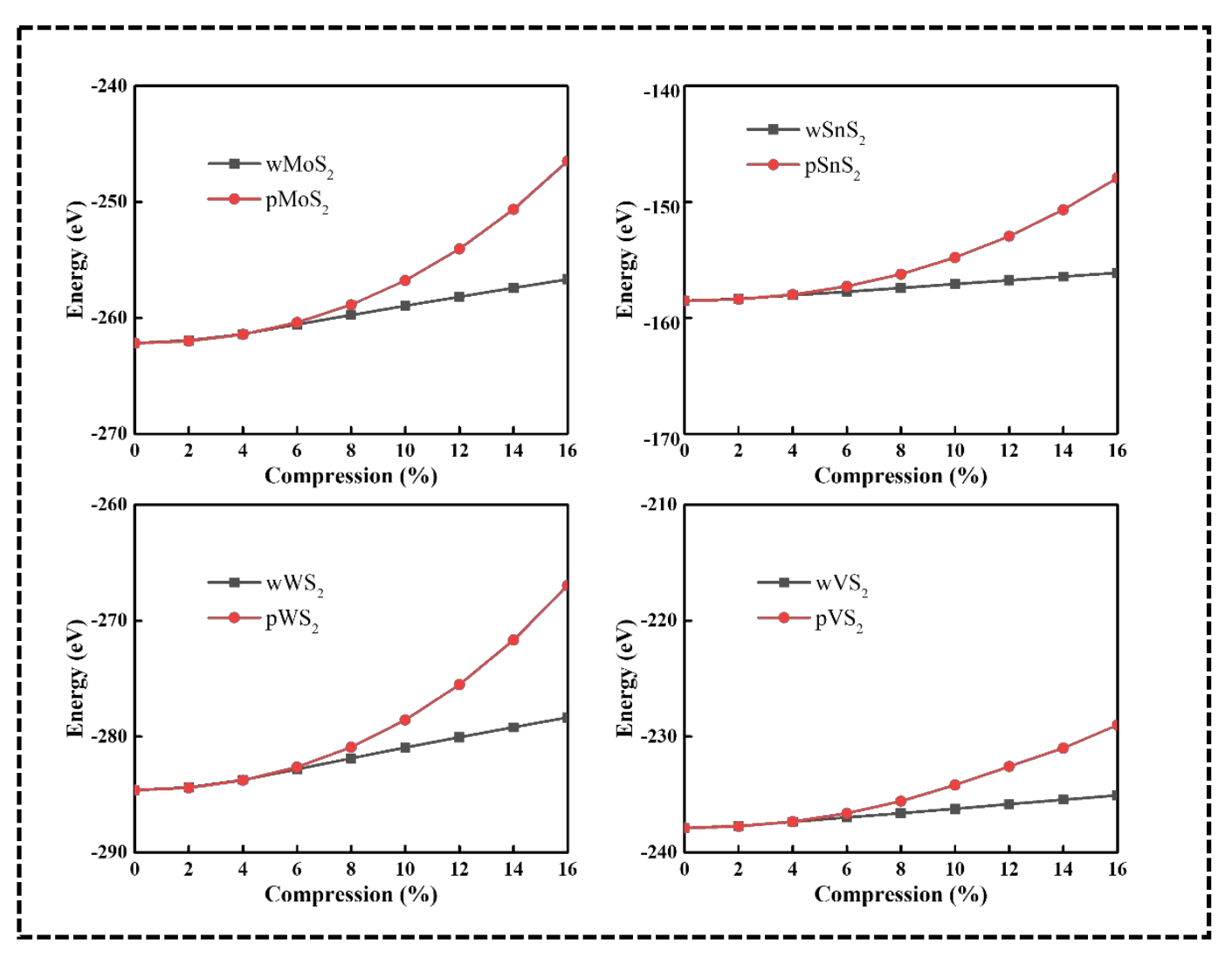

Figure S2. The calculated strain energies of $\mathrm{p}-\mathrm{MS}_{2}$ and $\mathrm{w}-\mathrm{MS}_{2}$ as a function of 
compression along the armchair direction.

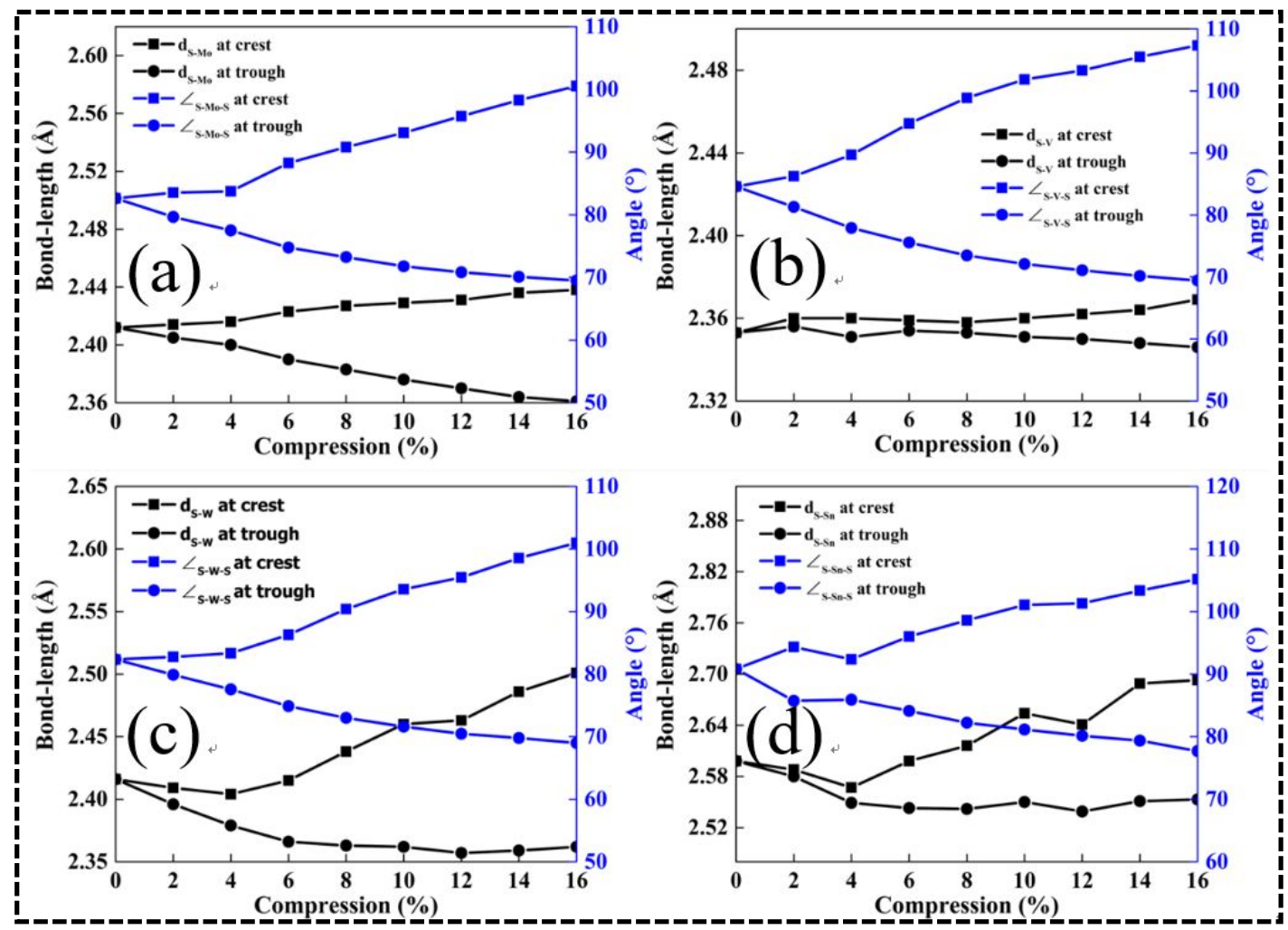

Figure S3. The calculated S-M bond length and S-M-S angle at crest and trough of waved $\mathrm{MS}_{2 .}$ (a) $\mathrm{MoS}_{2}$, (b) $\mathrm{VS}_{2}$, (c) $\mathrm{WS}_{2}$, and (d) $\mathrm{SnS}_{2}$. 


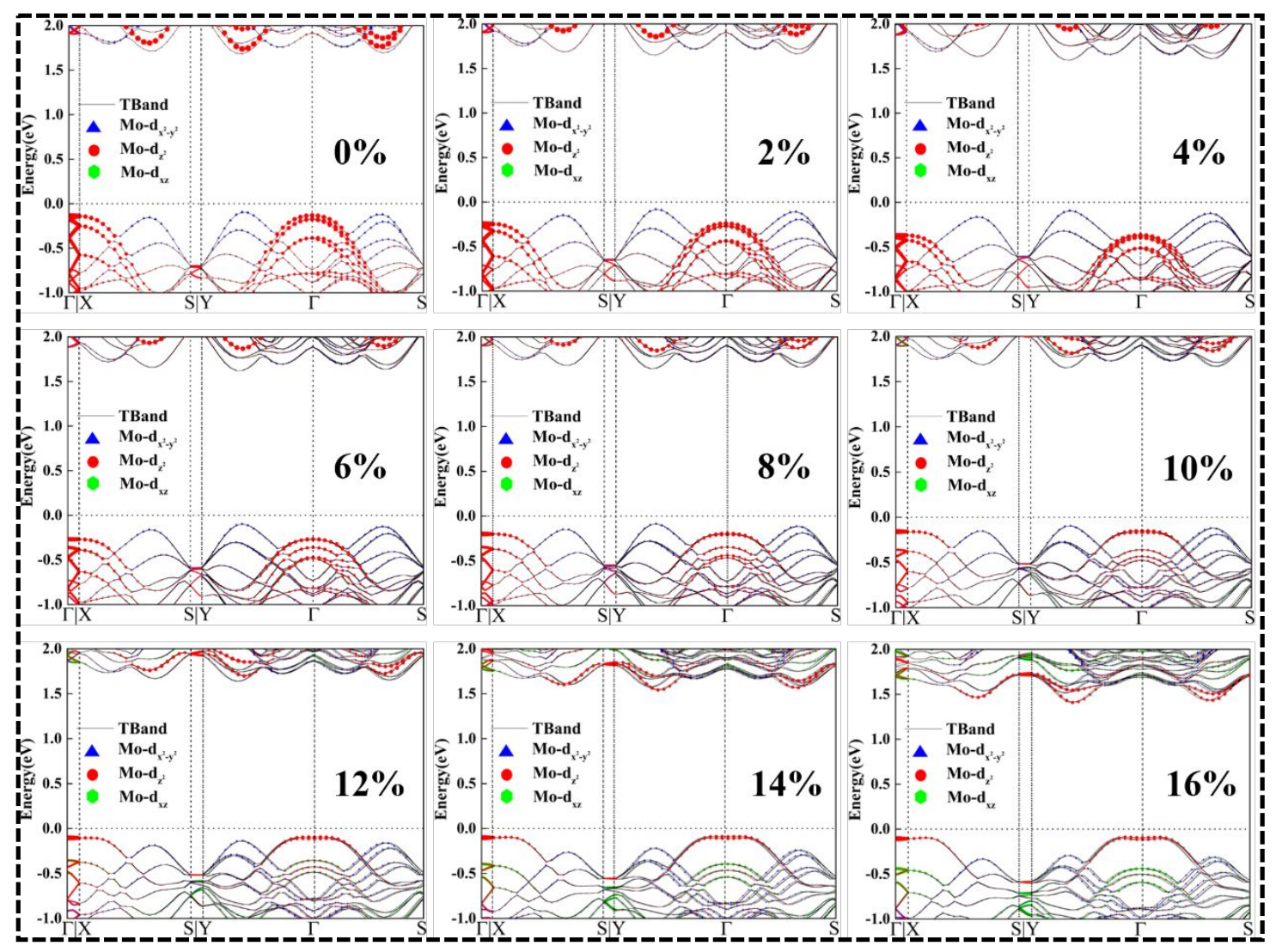

Figure S4. The projected band structures of $\mathrm{w}-\mathrm{MoS}_{2}$ under various

compressions from $0 \%$ to $16 \%$.

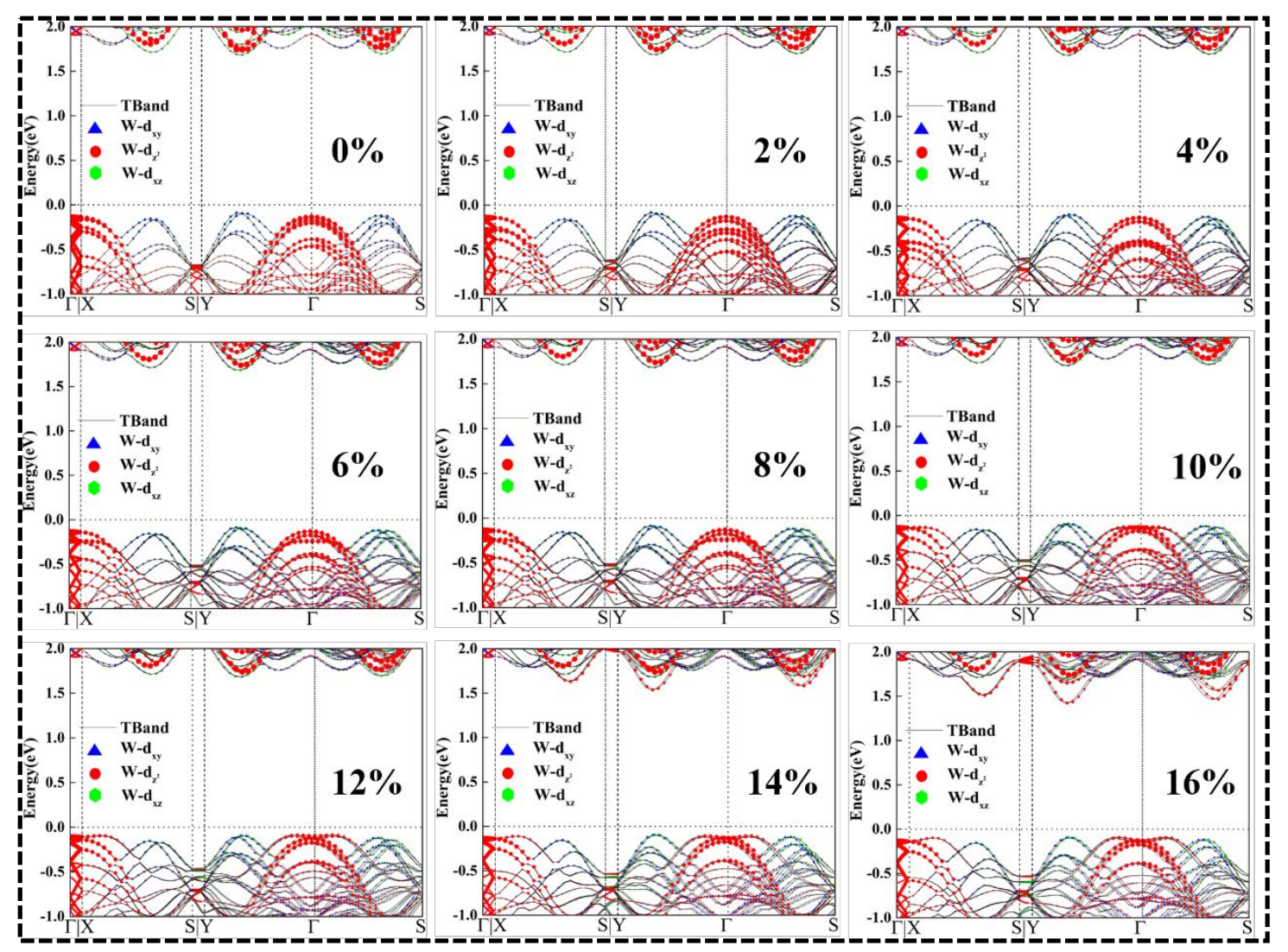


Figure S5. The projected band structures of $\mathrm{w}-\mathrm{WS}_{2}$ under various compressions from $0 \%$ to $16 \%$.

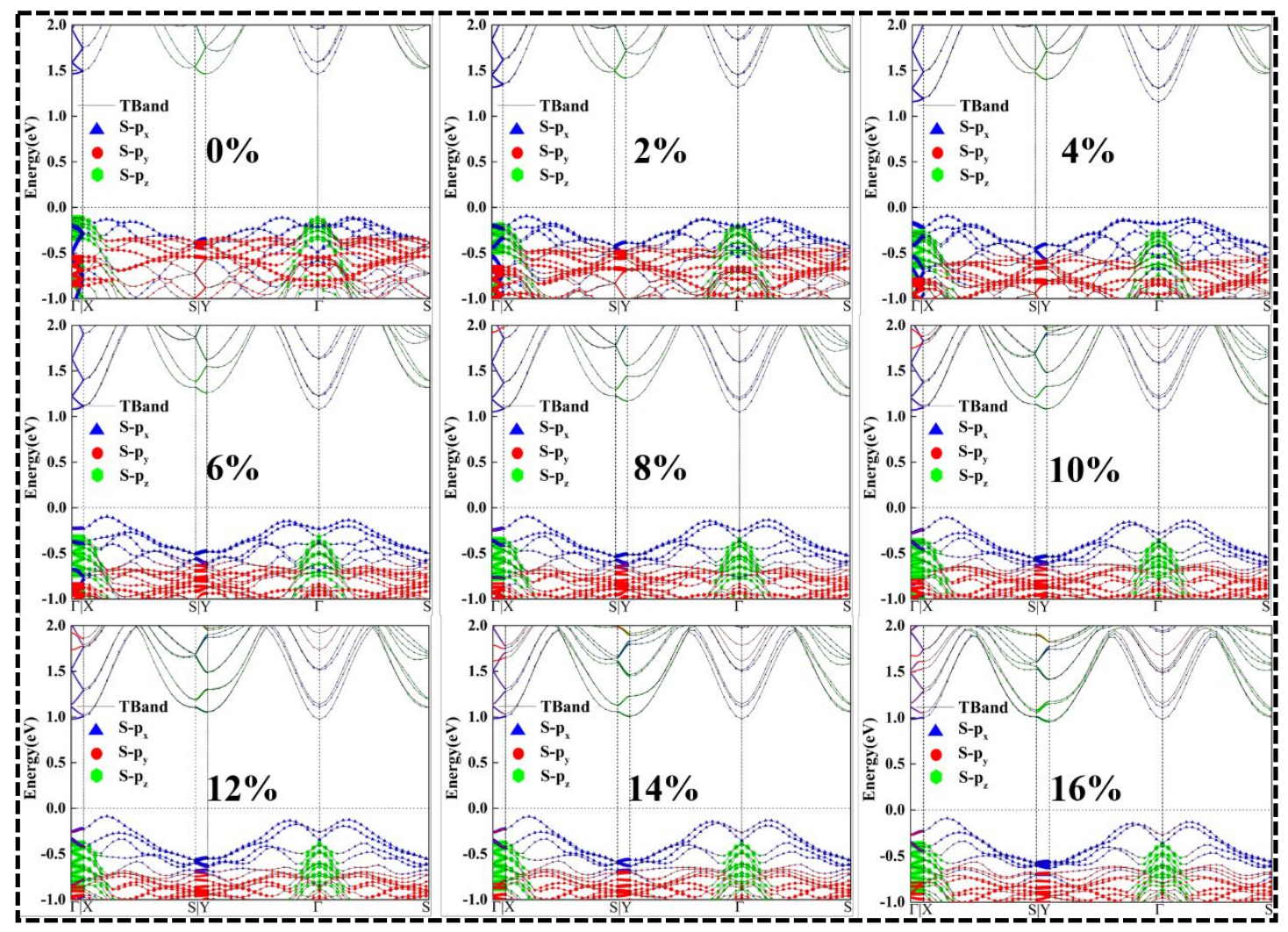

Figure S6. The projected band structures of $\mathrm{w}-\mathrm{SnS}_{2}$ under various compressions from $0 \%$ to $16 \%$. 


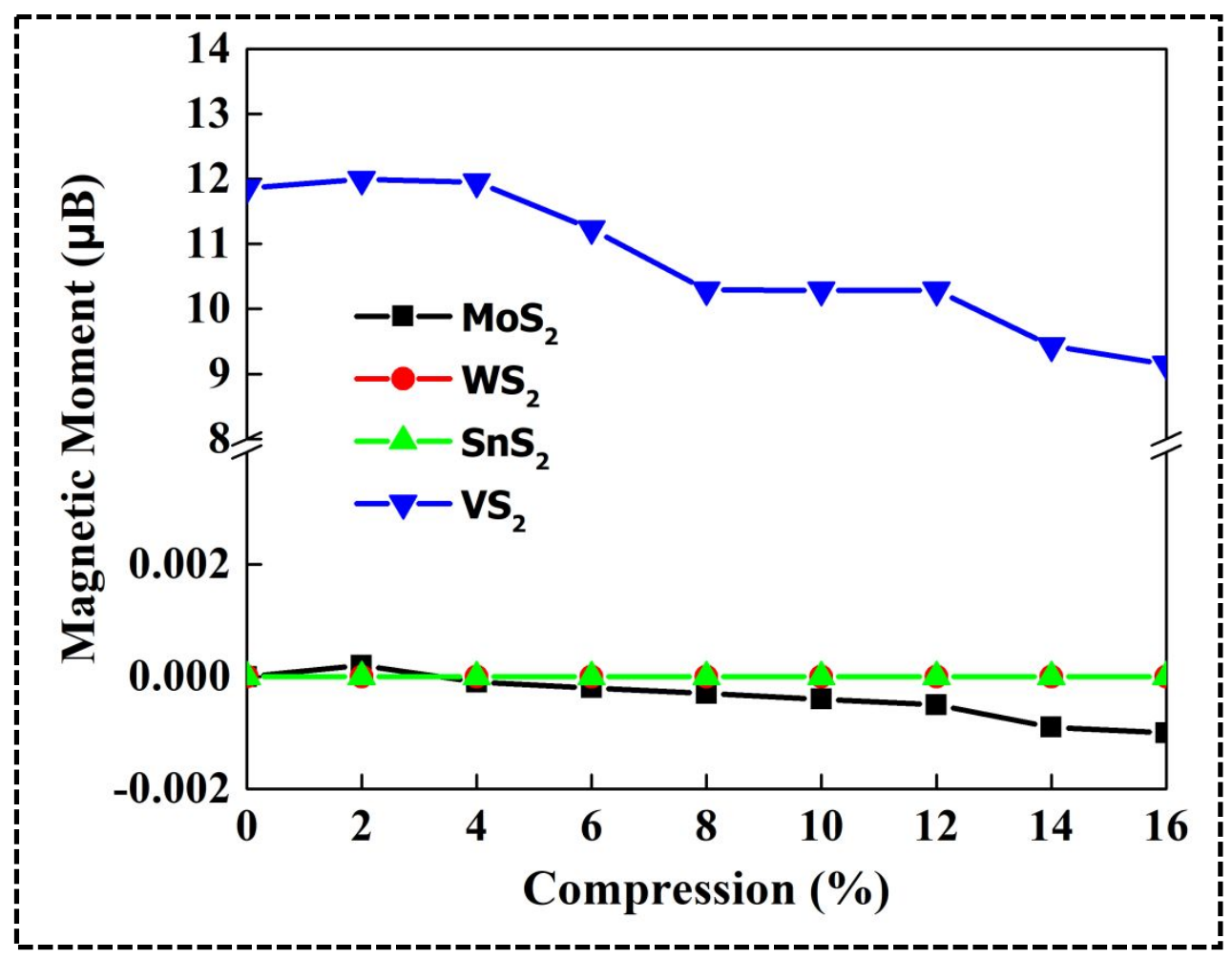

Figure S7. The calculated magnetic moments of $\mathrm{w}-\mathrm{MS}_{2}$ as a function of compression. 


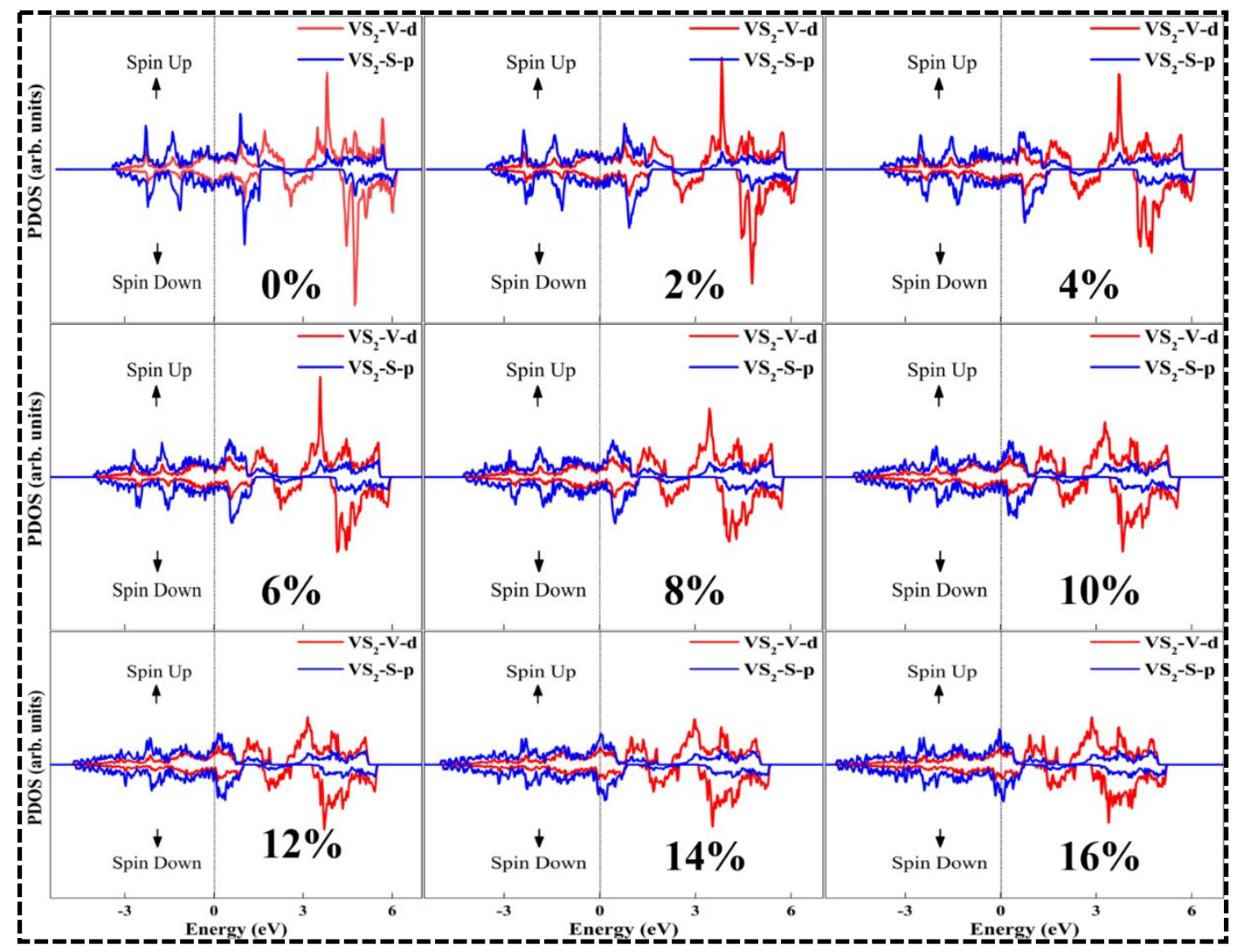

Figure S8. Density of states of $2 \mathrm{H} \mathrm{w}-\mathrm{VS}_{2}$ under different compressions.

Blue and red curves represent the PDOSs of different elements. The dish line stands for the Fermi level.

Figures S9-S10 show the relaxed structures of $\mathrm{w}-\mathrm{MS}_{2}$ with one $\mathrm{H}$ atom absorbed. We find that all the absorbed $\mathrm{H}$ tend to locate at the center position of honeycomb on the crest of $\mathrm{w}-\mathrm{MS}_{2}$ except for $\mathrm{TM}^{-\mathrm{MoS}_{2}}$ (Figure S9a \& e). The bond of $\mathrm{M}-\mathrm{H}$ increases as the compression increases (Table S1). The change of bonds of $\mathrm{M}-\mathrm{H}(\mathrm{S}-\mathrm{H})$ are from $1.434(1.719)$ to $1.371(1.723) \AA$, 1.366 (1.731) to 1.367 (1.865) $\AA, 1.408(1.721)$ to $1.364(1.703) \AA$, and $1.356(2.511)$ to 1.353 (2.536) $\AA$ for $w-W S_{2}, w-V S_{2}, w-M o S_{2}$, and $w-S n S_{2}$ with the increase of compression, respectively. Additionally, we see that the absorption of $\mathrm{H}$ results in breakdown of the $\mathrm{Sn}-\mathrm{S}$ bond on the crest of $\mathrm{w}-\mathrm{SnS}_{2}$ when $\sigma>12 \%$ (Figures 
S9d \& S10d), indicating its instability for HER at high curvature.

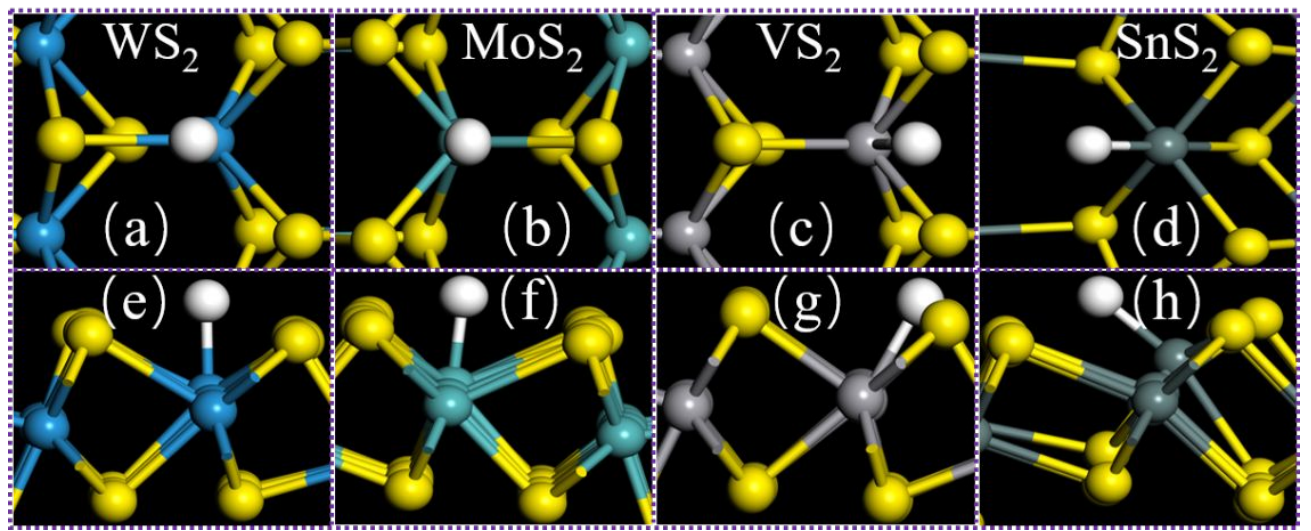

Figure S9. The relaxed $\mathrm{w}-\mathrm{MS}_{2}$ with $\mathrm{H}$ atom at $\mathrm{TM}$ at $\sigma=14 \%$ The upper row

represents the top view of TM site on $\mathrm{w}-\mathrm{MS}_{2}$. The nether row shows the related side views.

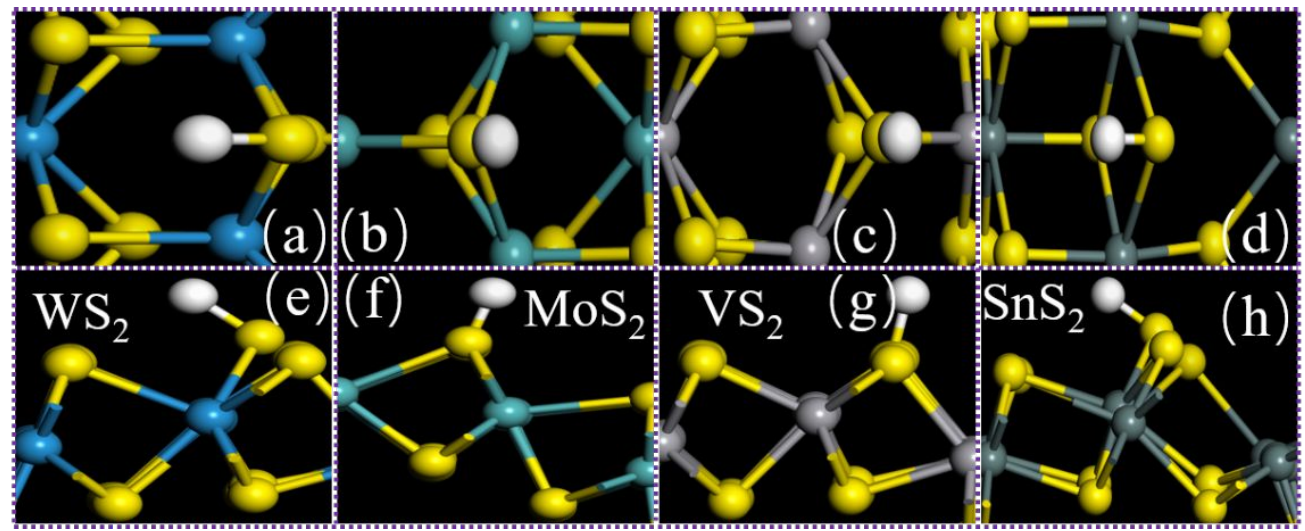

Figure S10. The relaxed $\mathrm{w}-\mathrm{MS}_{2}$ with $\mathrm{H}$ atom at $\mathrm{TS}$ at $\sigma=14 \%$ The upper row represents the top view of TS site on $\mathrm{w}-\mathrm{MS}_{2}$. The nether row shows the related side views. 


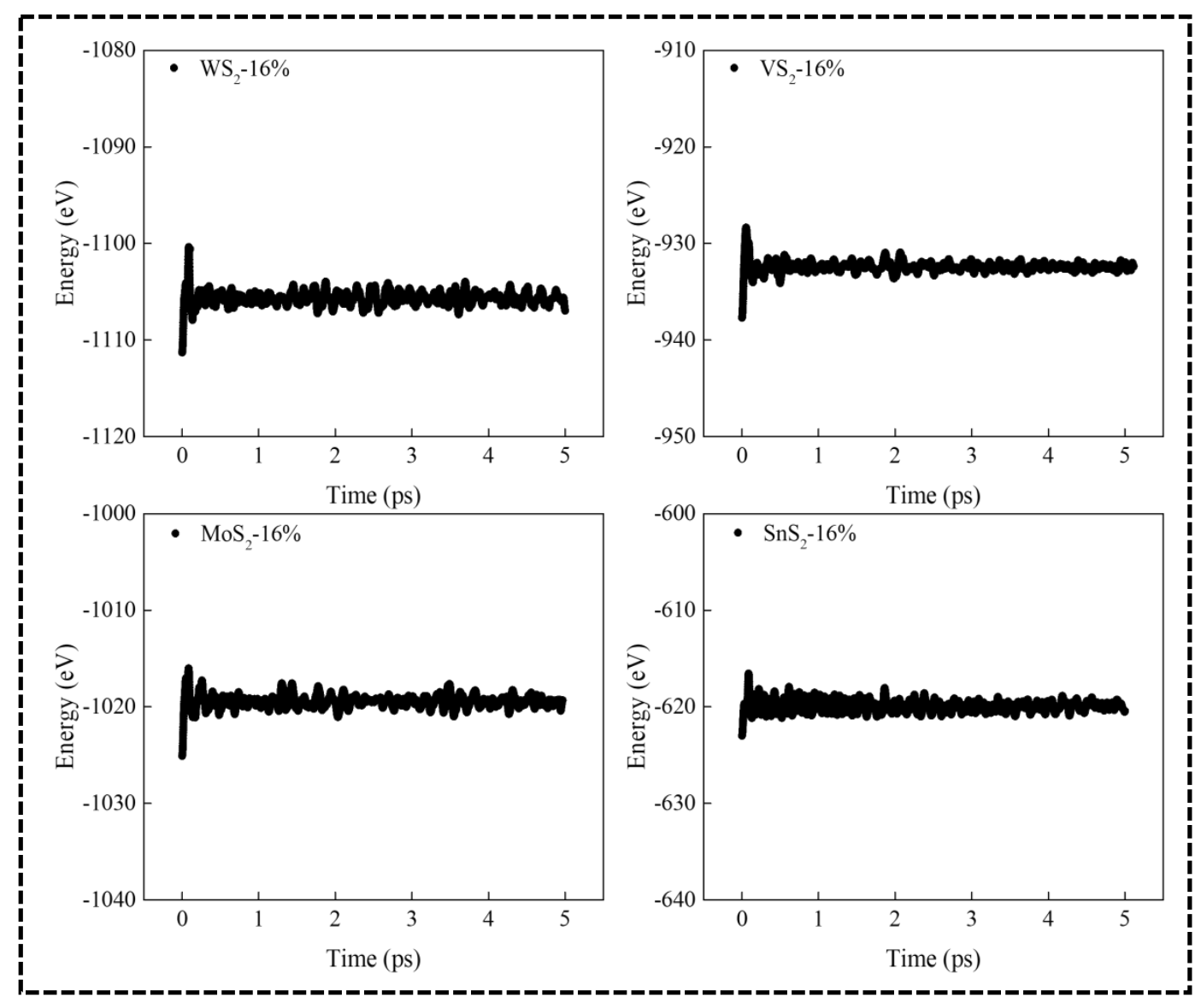

Figure S11. The molecular dynamics simulation of the total energy variations for $\mathrm{W}-\mathrm{MS}_{2}$ at $\mathrm{T}=300 \mathrm{~K}$. 


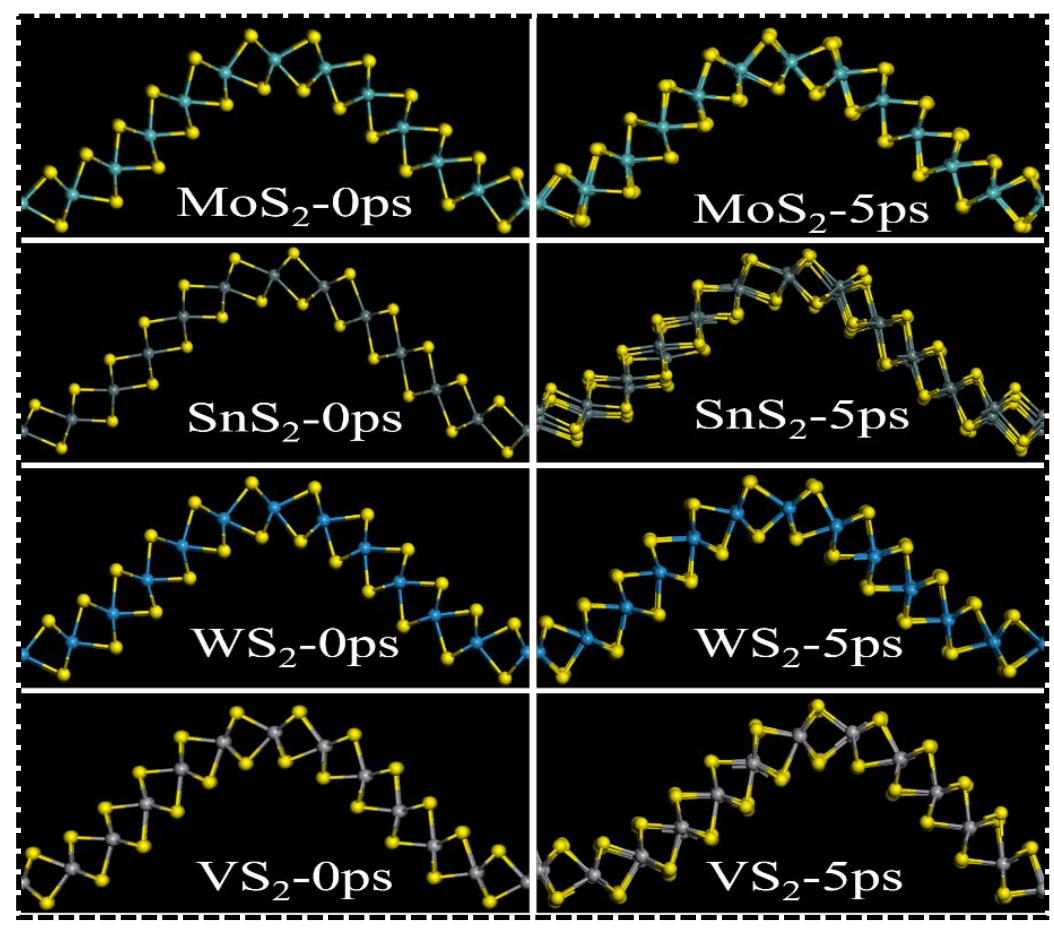

Figure S12. Snapshots of intermediates at different simulation times of w-

$\mathrm{MS}_{2}$. Left column: $0 \mathrm{ps}$; right column: $5 \mathrm{ps}$.

From the relaxed structures for $\mathrm{H}$ adsorbed at the $\mathrm{TM}$ site on $\mathrm{VS}_{2}$ with a compression from $4 \%$ to $14 \%$ (Figure S13), we see that the $\mathrm{H}$ atom tends to attach to the neighboring $\mathrm{S}$ atom when compression is less than $8 \%$. As the compression increases further, the effect from the $\mathrm{V}$ atom on the $\mathrm{H}$ adsorption is enhanced. Therefore, the adsorption of hydrogen atom on the TM site is not only affected by the metal atom, but the surrounding $S$ atoms because metal atoms are in the middle of the two $S$ layers $\left(\mathrm{MS}_{2}\right)$. The competitive effect from metal and surrounding $S$ atoms lead to different trends as a function of compression (Figure 7, $\mathrm{VS}_{2}$-TM). 


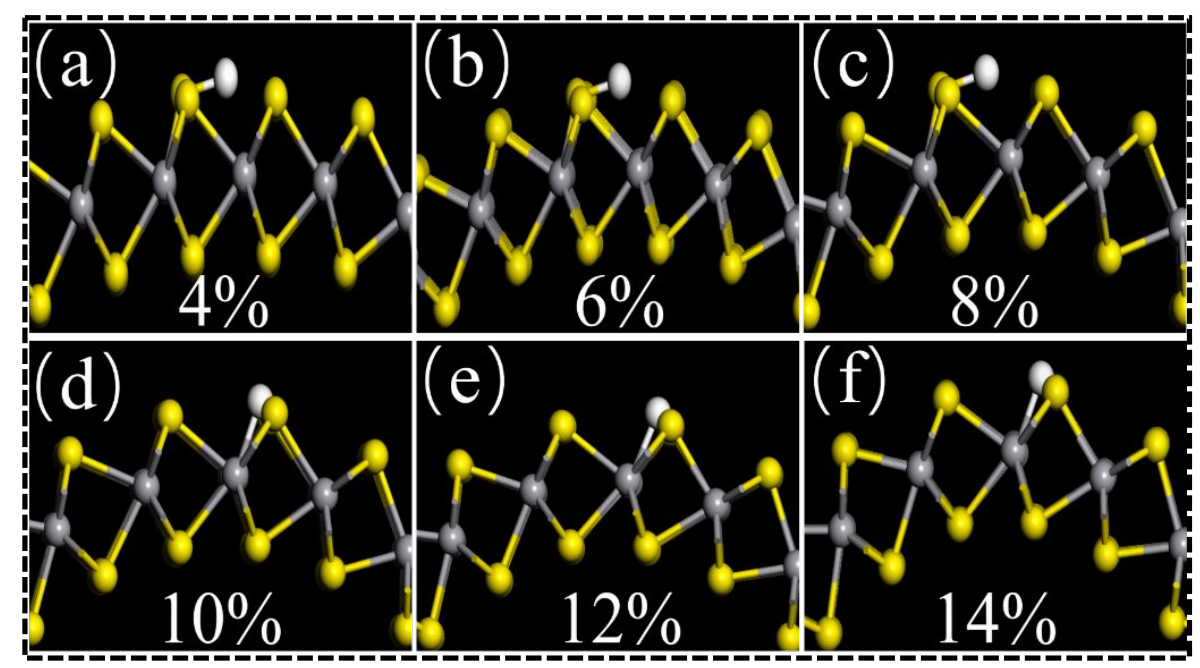

Figure S13. The relaxed $\mathrm{w}-\mathrm{VS}_{2}$ with $\mathrm{H}$ atom at $\mathrm{TM}$ from $\sigma=4 \%$ to $\sigma=14 \%$.

Table S1. The calculated S-H and $\mathrm{M}-\mathrm{H}$ bonds at different compressions.

\begin{tabular}{|c|c|c|c|c|c|c|c|c|}
\hline \multicolumn{9}{|c|}{ Bond Length $(\AA ̊)$} \\
\hline Monolayer & \multicolumn{2}{|c|}{$\mathrm{WS}_{2}$} & \multicolumn{2}{|c|}{$\mathrm{VS}_{2}$} & \multicolumn{2}{|c|}{$\mathrm{MoS}_{2}$} & \multicolumn{2}{|c|}{$\mathrm{SnS}_{2}$} \\
\hline Compressio & $\mathrm{S}-\mathrm{H}$ & $\mathrm{M}-\mathrm{H}$ & $\mathrm{S}-\mathrm{H}$ & $\mathrm{M}-\mathrm{H}$ & $\mathrm{S}-\mathrm{H}$ & $\mathrm{M}-\mathrm{H}$ & $\mathrm{S}-\mathrm{H}$ & $\mathrm{M}-\mathrm{H}$ \\
\hline $\mathrm{n}(\%)$ & & & & & & & & \\
\hline 0 & 1.434 & 1.719 & 1.366 & 1.731 & 1.408 & 1.721 & 1.356 & 2.511 \\
\hline
\end{tabular}




\begin{tabular}{llllllllll}
\cline { 2 - 8 } 2 & 1.442 & 1.714 & 1.364 & 1.911 & 1.407 & 2.310 & 1.356 & 2.515 \\
\cline { 2 - 9 } 4 & 1.431 & 1.714 & 1.365 & 1.901 & 1.409 & 1.713 & 1.356 & 2.495 \\
\cline { 2 - 9 } 6 & 1.425 & 1.711 & 1.365 & 1.932 & 1.400 & 1.698 & 1.354 & 2.523 \\
\cline { 2 - 9 } 8 & 1.403 & 1.717 & 1.366 & 1.934 & 1.399 & 1.695 & 1.354 & 2.533 \\
\cline { 3 - 9 } 10 & 1.393 & 1.716 & 1.367 & 1.869 & 1.399 & 1.699 & 1.353 & 2.536 \\
14 & 1.382 & 1.718 & 1.368 & 1.870 & 1.394 & 1.698 & 1.354 & 2.539 \\
\hline 16 & 1.371 & 1.723 & 1.367 & 1.865 & 1.364 & 1.703 & Broken & Broken \\
\cline { 2 - 8 } & & 1.720 & 1.368 & 1.864 & 1.363 & 1.701 & Broken & Broken \\
\hline
\end{tabular}


Table S2. The calculated net Bader charge for $\mathrm{w}-\mathrm{MS}_{2}$ with $\mathrm{H}$ atom at TS under different compressions.

$\mathrm{H}$ charge transfer (e)

\begin{tabular}{ccccc}
\hline Compression & $\mathrm{WS}_{2}$ & $\mathrm{VS}_{2}$ & $\mathrm{MoS}_{2}$ & $\mathrm{SnS}_{2}$ \\
$(\%)$ & & & & \\
0 & 0.006 & -0.047 & -0.020 & -0.192 \\
2 & 0.034 & -0.055 & -0.069 & -0.191 \\
4 & 0.005 & -0.059 & -0.030 & -0.192 \\
6 & 0.067 & -0.046 & 0.035 & -0.191 \\
8 & 0.090 & -0.051 & 0.055 & -0.192 \\
10 & 0.111 & -0.086 & 0.079 & -0.184 \\
12 & 0.127 & -0.070 & 0.080 & -0.184 \\
14 & 0.157 & -0.045 & 0.108 & Broken \\
16 & 0.162 & -0.077 & 0.121 & Broken \\
\hline
\end{tabular}

